\title{
PROCESOS SEDIMENTARIOS Y DIAGENÉTICOS RECIENTES Y SU IMPORTANCIA COMO FACTORES DE INTERPRETACIÓN DE SUS ANÁLOGOS ANTIGUOS
}

\author{
Joaquín Eduardo Aguayo Camargo*
}

\begin{abstract}
RESUMEN
La sedimentología como investigación científica y aplicada, ha tenido un importante impulso durante las tres últimas décadas, debido a las implicaciones que tiene en la identificación y análisis de las provincias tectono-sedimentarias recientes, continentales y oceánicas, cuyo entendimiento sirve como base para interpretar a sus análogos antiguos, los que, en conjunto, conforman a la columna estratigráfica. El tema es sumamente extenso y existe una literatura temática amplia, por lo que en este trabajo se hace especial énfasis en las rocas calcáreas y terrígenas por ser éstas importantes en la acumulación de hidrocarburos y de minerales. En particular las calizas dolomitizadas contienen un volumen considerable de aceite y gas y conforman espesores de varios cientos de metros, difícilmente interpretables geológicamente; por lo que se mencionan algunas técnicas utilizadas como rutina en otros países, adaptadas al microscopio petrográfico convencional, revelándose las partículas y texturas originales de las rocas, lo que facilita la interpretación de los ambientes sedimentarios y subambientes; Los nuevos criterios sedimentológicos abren una frontera de investigación en la historia evolutiva de las provincias geológicas en particular de aquellas cuya columna estratigráfica está dolomitizada y que son altamente petrolíferas.
\end{abstract}

\begin{abstract}
The sedimentology has had a very important impulse during the last three decades, as science and as applied research, due to its implications on the identification and analysis of the recent continental and oceanic tectono-sedimentary provinces; its understanding is the base to identify and interpret their ancient analogous, which, as whole, conform the stratigraphic column. The subject is so wide and actually exist a broad literature about it; therefore, in this paper is only taken in account the calcareous and terrigenous rocks because they are important as reservoirs of hydrocarbons and minerals. Particularly the dolomitized limestones have considerable volume of oil and gas and have hundreds of meters of thickness in the stratigraphic column, such are very difficult to interpret geologically. Therefore, also are mentioned some techniques used as routine in other countries that are adapted to the conventional petrographic microscope to show the original particles and textures of the rocks and give some light to interpret the sedimentary environments and subenvironments. The new sedimentological criteria open a frontier to the research about the evolutive history of the geological provinces, particularly in such ones in which the stratigraphic column is dolomitized and are highly petroliferous.

*Instituto de Ciencias del Mar y Limnología, UNAM
\end{abstract}




\section{INTRODUCCIÓN}

La geología ha tenido un despertar vertiginoso en los últimos 25 años, después de haber pasado por un letargo prolongado de varias décadas. Este nuevo impulso se debió, fundamentalmente a la revolución de ideas que causó la postulación de la teoría sobre la expansión del fondo oceánico y su relación con el desplazamiento de los continentes, ideas propuestas por H. H. Hess (1962), R. S. Dietz (1961), quienes plantearon que los movimientos tectónicos regionales eran el resultado de una repercusión global y no se debían simplemente a inestabilidades locales de la litosfera. Por otra parte, existía la inquietud de hacer de la geología una ciencia más cuantitativa, sin perder sus matices subjetivistas, que son la observación misma de los fenómenos naturales y de la interpretación de las causas que los originan, ya que éstos no son fácilmente cuantificables debido a su complejidad. La identificación e interpretación de los procesos geológicos dependen de la experiencia, persistencia y curiosidad de los estudiosos de las Ciencias de la Tierra que tratan de entender la evolución de nuestro planeta y del Universo mismo.

Por coincidencia se han conjuntado varios aspectos en la postulación de la teoría de Tectónica Global; por un lado, la tecnología, en todas sus fases, avanzó a pasos agigantados, y por el otro, la necesidad mundial de localizar recursos minerales particularmente energéticos; propició que las grandes empresas petroleras de los países altamente desarrollados fomentaran la investigación básica y la aplicada, en todas las áreas de la ciencia geológica.

En lo particular, sólo se hará referencia sobre conceptos de los sedimentos y de las alteraciones físico-químicas y bioquímicas que sufren los mismos a partir de su depósito hasta su sepultamiento final, procesos conocidos como diagenéticos,

Numerosos grupos de investigadores revisaron los conceptos sobre modelos sedimentarios, elaborados en décadas anteriores y analizaron simultáneamente los procesos geológicos que actúan en las rocas que afloran en el continente y que son las principales fuentes de generación de sedimentos; de esta manera pudieron determinarse y cuantificarse los parámetros que gobiernan el transporte y el depósito de las partículas sedimentarias, así como el grado de destrucción de los diferentes minerales y fragmentos rocosos bajo diversas condiciones de clima y de energía. Con base en estos conceptos se hicieron múltiples observaciones en los ambientes recientes de depósito, complementándose las realizadas en laboratorios de sedimentología y de geoquímica en universidades e institutos de investigación de varios países.

Los investigadores describieron con detalle las facies sedimentarias que conforman los ambientes de depósito bajo diversas condiciones tectónicas y climáticas, desde continentales hasta litorales y desde marinas someras hasta profundas. Estas últimas son exploradas por barcos oceanográficos equipados con instrumentos de medición de alta precisión 
analítica y con equipos de muestreo técnicamente sofisticados; tal es el caso, en México, de los estudios hechos por el "Glomar Challenger del Deep Sea Drilling Project", que recabó información del subsuelo de las cuencas oceánicas del Pacífico y del Golfo de México. También se fotografiaron los fondos marinos del Océano Pacífico y del Golfo de California; se colectaron sedimentos directamente por medio de submarinos, como el "Alvin" y el "Cyana", propiedad de Estados Unidos y de Francia respectivamente. Nos referimos a ellos como un simple ejemplo, ya que existen varios barcos oceanográficos y son numerosas las expediciones que se realizan anualmente con buques oceanográficos nacionales como el "Mariano Matamoros"' el "Puma" y el "Justo Sierra", entre otros más que, exploran las regiones del Pacífico, del Golfo de México y del Caribe, para inventariar y evaluar los recursos naturales no renovables que existen en la zona exclusiva del mar patrimonial de México.

En síntesis, a partir de la década de los sesenta, los criterios de análisis e interpretación de los ambientes sedimentarios han sido diferentes de los de las décadas anteriores y, por ende, la columna estratigráfica está siendo reinterpretada nuevamente, ya que es parte fundamental de la ciencia geológica para entender los eventos dinámicos a los que ha estado sujeto nuestro planeta a través de toda su historia evolutiva. Debemos estar conscientes de que la postulación de la teoría de Tectónica Global no es por si sola la "piedra filosofal" que convierte todo en respuesta; sin embargo, es oportuno señalar que ha dado la pauta para hacer una revisión profunda sobre las ideas que tradicionalmente se consideraban como "ampliamente" conocidas y "satisfactoriamente" explicadas; puesto que las interpretaciones simples sobre la geodlnámica mundial son inadecuadas en la medida que se enriquece el conocimiento científico con la obtención de nueva información, o bien, cuando los datos previos se organizan y reinterpretan a la luz de los criterios que dicta el estado actual de la ciencia.

\section{AMBIENTES SEDIMENTARIOS Y ANÁLISIS DE FACIES}

Los sedimentos, como partículas individuales o como agregados, son el producto de las interacciones complejas entre la corteza terrestre y la atmósfera, la hidrosfera y la biosfera; procesos que son multiplicados por el tiempo geológico. Como sabemos, la corteza terrestre, está constituida por rocas antiguas que nos revelan toda la historia previa de la evolución tectónica y sedimentaria que ha sufrido el planeta. Al ser expuestas en la superficie estas rocas sufren, como todo producto físico-químico fuera de su ambiente de formación, el ataque de los agentes atmosféricos y orgánicos y buscan equilibrarse bioquímicamente con el nuevo ambiente que los rodea. La incidencia de los rayos solares sobre la superficie del terreno, la fisiografía del mismo, la latitud y la altitud, son algunos de los factores que regulan el clima. 
Podemos agrupar los productos minerales resultantes de la desintegración de las rocas preexistentes en cuatro tipos generales:

1) minerales que resistieron al ataque de los agentes físico-químicos y biológicos y que se conservan como partículas disgregadas;

2) minerales que se hidratan $y$ tienden $a$ pulverizarse hasta tamaños de limo y arcilla;

3) minerales de neoformación, productos de la disolución parcial de la roca y de la precipitación in situ de estas soluciones a nuevos minerales.

4) minerales derivados de la disolución de la roca original, y que se precipitan en una cuenca sedimentaria fuera de los sitios de los que provienen.

Los cuatro productos minerales son acarreados finalmente hasta el mar, en donde sufren nuevamente una serie de transformaciones mediante procesos orgánicos e inorgánicos, hasta equilibrarse con el nuevo ambiente que los controla. Los iones aportados al mar son sintetizados por los organismos lacustres y marinos; algunos de ellos son atrapados en estructuras rígidas como testas y conchas; otros iones son retenidos en el cuerpo blando de los organismos y cumplen la función de agentes metabolizantes; otros más, son atrapados inorgánicamente en las partículas arcillosas y en los minerales precipitados físico-químicamente.
Sabemos que los procesos de intemperismo, erosión y transporte, que actúan sobre los continentes, juegan un papel muy importante en el ciclo evolutivo de la vida y en el depósito de sedimentos en las cuencas oceánicas. Se ha calculado que el aporte actual anual de sedimentos al mar por erosión del continente es aproximadamente de $60 \mathrm{Km}^{3}$ (Armstrong, 1969); sin embargo, un gran volumen de sedimentos se deposita en las plataformas durante varios cientos de años. Posteriormente, a causa de los movimientos sísmicos o bien por la carga excesiva de los sedimentos, éstos se desplazan hacia el fondo de las cuencas oceánicas como una simple avalancha mezclada con el agua marina y transportan volúmenes del orden de 0.01 a $100 \mathrm{Km}^{3}$ (Walker, 1973); lo anterior se ha observado en la columna estratigráfica, bajo la forma de depósitos turbidíticos (sedimentos transportados en condiciones subacuosas por corrientes de turbidez).

Durante su deslizamiento, estas grandes avalanchas submarinas, erosionan a su vez el subestrato marino; el volumen erosionado es de gran magnitud: del orden de 300 a $450 \mathrm{Km}^{3}$, como se calculó en los cañones submarinos Delgado y Monterrey en el borde continental de California, en la costa suroccidental de Estados Unidos. Este gran volumen de sedimentos se depositó paulatinamente al pie del talud continental formando abanicos abisales que contienen entre 300,000 y $450,000 \mathrm{Km}^{3}$ de sedimentos acumulados (Menard, 1964).

Los mecanismos de transporte y depósito de las partículas sedimentarias dependen del tipo, de la 
velocidad y de la persistencia de las corrientes que los gobiernan, ya sean eólicas, fluviales o marinas. Una vez que ocurre el depósito, los sedimentos son sepultados por otros $y$ nuevamente sufren transformaciones físico- químicas al reaccionar con los fluidos intersticiales contenidos entre las partículas sedimentarias; estas reacciones modifican las condiciones de oxidación-reducción y de acidezalcalinidad del ambiente de depósito, pues hacen variar la salinidad, temperatura y presión en el mismo. Los procesos mencionados litifican a las partículas disgregadas, ya sea mediante la compactación, la cementación, o bien ambos fenómenos; también las recristalizan, las disuelven, o son reemplazadas iónicamente por otros minerales de neoformación o autigénicos. A este conjunto de reacciones interactivas, que llegan a enmascarar los rasgos texturales primarios del sedimento original se le conoce como Proceso diagenético, de él hablaremos posteriormente.

Los sedimentos contenidos en la columna estratigráfica no sólo son el producto de la desintegración de las rocas que afloran en el continente, del transporte y del depósito de los sedimentos resultantes, como fue el caso expuesto; también se genera in situ un gran volumen de partículas de carbonato de calcio y grandes extensiones de evaporitas (sulfatos y cloruros), principalmente en los litorales y sobre la plataforma marina continental, actualmente entre las latitudes $30^{\circ}$ norte y $30^{\circ}$ sur. Estas rocas carbonatadas y evaporíticas son abundantes desde el Paleozoico y han sido tema de investigación desde hace dos o tres siglos. Sin embargo, fue hasta mediados del siglo pasado (1853), cuando el inglés Henry C. Sorby, hizo el primer estudio petrográfico cuantitativo de las calizas y detalló sistemáticamente la dirección de paleocorrientes en estratos antiguos; además estableció las bases para reconocer las partículas principales que constituyen este tipo de rocas y reconoció además los ambientes sedimentarios que las originan. Antes de Sorby, los paleontólogos y estratígrafos desconocían con detalle los ambientes de sedimentación que originaban a las rocas sedimentarias, como él lo observó.

Sin embargo, fue hasta un siglo después, cuando las agrupaciones científicas de las Ciencias de la Tierra sintieron la necesidad de difundir con mayor profundidad los conocimientos sobre el origen de las calizas, y de interpretar sus estructuras internas y texturas; esto pudo ser posible gracias al aliento y apoyo económico proporcionado por las grandes empresas petroleras, ya que de estos estratos calcáreos se estaban extrayendo gran cantidad de hidrocarburos entrampados tanto en estructuras geológicas tales como anticlinales y fallas, como en los cambios de litofacies laterales, conocidos como trampas estratigráficas, que no tienen ninguna expresión estructural aparente. Aún más, también encontraban petróleo y gas en las calizas sin cambios de litofacies laterales y sin expresión estructural pero con cambios de permeabilidad lateral y vertical; a éstas se les denomina trampas diagenéticas. 
En vista de los nuevos descubrimientos petrolíferos, en 1959, "The American Association of Petroleum Geologists" (A.A.P.G), "The Society of Economic Paleontologists and Mineralogists" (S.E.P.M.), auspiciaron la reunión de un grupo de científicos para que revisaran las clasificaciones de calizas que se habían propuesto y utilizado durante más de un siglo. Los sistemas de clasificación se discutieron durante dos años y los resultados fueron publicados en 1962 en la Memoria -1 de The American Association of Petroleum Geologists: "Classification of Carbonate Rocks", y cuyo editor fue William E. Ham; la clasificación de las calizas fue el punto de partida fundamental para lograr un mejor entendimiento sobre los procesos que las originan y sobre los mecanismos geológicos que las preservan o destruyen. De las clasificaciones propuestas, que en la actualidad son más de 12 , solamente dos han sido ampliamente aceptadas y utilizadas por la comunidad geológica mundial: la propuesta por Robert L. Folk, y la de Robert J. Dunham. La clasificación del primer autor es composicional y textural, y distingue cinco constituyentes mayores o principales, de los cuales, a cuatro los agrupa y los denomina como "aloquímicos " (origen no químico):

1) fragmentos fósiles

2) oolitas y pisolitas

3) fragmentos de sedimentos retrabajados o intraclastos $y$
4) pellas de posible origen fecal formados por la excreción de crustáceos, anélidos y otros organismos que viven en lagunas litorales, planicies de mareas y plataformas marinas someras.

El quinto constituyente calcáreo formador de calizas está constituido por los grandes crecimientos orgánicos coloniales, como algas, corales, esponjas, rudistas y otros fósiles también coloniales que han desaparecido a través del tiempo geológico, y cuya particularidad es la de formar complejos arrecifales, ya que estos organismos son, o fueron, sedentarios; por esta razón los agrupó con el término de "Biolititas" (bios: vida; litos: piedra). Tanto las partículas aloquímicas como los arrecifes, están litificados por otros dos constituyentes: por una matriz constituida de fango calcáreo que se infiltra físicamente entre los poros de los aloquímicos, y por un cemento calcáreo que también está contenido entre los poros de los sedimentos, pero que precipita espontáneamente a partir de una solución saturada de carbonato de calcio, ya sea como calcita espática con bajo contenido de magnesio, como aragonita, o bien como calcita hipermagnesiana. R.L. Folk (op. cit.) denominó a estos dos constituyentes como "ortoquímicos" (precipitados, químicos).

La clasificación de R. J. Dunham es textural y no propone alguna terminología en particular para los diferentes tipos de aloquímicos que no fueran los mencionados por Folk. Su clasificación se 
basa en la relación que existe entre la matriz y el cemento con las partículas calcáreas o aloquímicos. El autor separa, al igual que R.L. Folk, los crecimientos orgánicos coloniales formadores de arrecifes y los denominan Boundstone (sin traducción al español).

La postulación de las dos clasificaciones despertó gran interés y entusiasmo por conocer la génesis y distribución de los constituyentes calcáreos en los innumerables ambientes recientes de depósito de todo el mundo, en diversas latitudes. Grupos de sedimentólogos volvieron a revisar aquellas provincias sedimentarias de Bahamas, Florida, Belice, Cancún, Isla Mujeres. y otras no tradicionales por inaccesibles, como las de Australia, India, y las islas de los mares del Pacífico, del Caribe y del Atlántico.

El volumen de los trabajos publicados sobre ambientes sedimentarios y del análisis de sus facies es numeroso, al igual que las revistas científicas especializadas que escriben sobre el tema. Es de esperar que la enorme producción científica publicada en varios idiomas durante las dos últimas décadas es difícil de asimilar, por lo que se ha venido agrupando temáticamente en:

1) Glosarios ilustrados con fotografías de las partículas orgánicas e inorgánicas, y texturas primarias y secundarias fotografiadas en el microscopio petrográfico convencional y con el de barrido electrónico.
2) Glosarios fotográficos de estructuras sedimentarias y primarias.

3) Publicaciones temáticas de ambientes sedimentarios específicos y de las facies que los caracterizan.

Este cúmulo de conocimientos es fundamental para distinguir e interpretar los depósitos sedimentarios análogos antiguos que constituyen a las rocas de la columna estratigráfica. Para poder predecirlas en el subsuelo es necesario tener un buen conocimiento sobre los mecanismos que las originan $y$ de su distribución en el tiempo y en el espacio. Aún así, existen ciertas limitaciones, ya que comúnmente no se cuenta con el muestreo apropiado y la columna estratigráfica está estructuralmente deformada de manera que las rocas que la constituyen se han alterado parcialmente. es decir, diagenéticamente; aún más, sus características petrológicas y primarias han desaparecido completamente por efectos de la alta presión y temperatura a las que han sido sometidas a través del tiempo; a este fenómeno se le conoce como metamorfismo.

\section{DIAGÉNESIS Y POROSIDAD}

El término de diagénesis (conocido también como metasomatismo), fue acuñado por $\mathrm{J}$. Walther en 1893-94, (en: Wolf y Chilingarian, 1976), al referirse a los cambios físico-químicos que sufren los sedimentos durante el sepultamiento, y que concluyen con su 
litificación en condiciones bajas de temperatura y presión; aún así, se conservan, o son discernibles su características texturales, estructurales y mineralógicas primarias, a pesar de que estas últimas hayan sufrido procesos de recristalización, reemplazamiento o disolución parcial.

Es importante identificar las fases sucesivas de alteración físico-química y biológica que han sufrido las partículas, o el conjunto de sedimentos, desde que se depositan hasta que se litifican y posteriormente a ello, pues de esta manera es posible reconstruir conjuntamente con otros parámetros tales como los estructurales y estratigráficos la historia tectónica evolutiva de las provincias geológicas. Es decir, entendiendo la evolución diagenética también conocida como paragénesis se pueden predecir los estratos con diferencias de permeabilidad en el subsuelo, dicho proceso es necesario en las exploraciones de yacimientos minerales y de hidrocarburos. El principal motivo por el que las grandes empresas petroleras apoyaron estudios de esta índole, fue la perspectiva de llegar a comprender las causas de la porosidad y permeabilidad de las rocas sedimentarias que sirven como receptáculos al petróleo y al gas para poderlos localizar en el interior de la corteza terrestre.

A mediados del siglo pasado H.C. Sorby, como ya se mencionó, reconoció texturas de recristalización, disolución y reemplazamiento en rocas calcáreas. así como otras que son el producto de la transformación de polimorfos minerales (minerales de la misma composición pero con estructura cristalina diferente); tal es el caso del neomorfismo que sufre la aragonita al convertirse en calcita, ambas están constituidas por carbonato de calcio, pero la primera cristaliza en el sistema ortorrómbico (hábito fibroso) y la segunda en el hexagonal (hábito en bloque). Más tarde Cullis (1904) estableció la secuencia paragenética de cementación, recristalización y reemplazamiento en dolomita a partir de la aragonita y de la calcita. Otros investigadores trabajan aisladamente con estas ideas, pero sin entender la importancia que tienen los procesos de diagénesis en la historia evolutiva o petrogenética de las secuencias estratigráficas. Fue hasta los años cincuenta cuando Graf y Lamar (1950), Bergenback y Terriere (1953), Bathurst $(1958,1959)$, Folk $(1959,1965)$ y Wolf $(1965)$, entre otros, trataron de dilucidar criterios para distinguir la calcita espática derivada de la recristalización del fango calcáreo, de la calcita espática precipitada espontáneamente como cemento, a partir de una solución saturada en carbonato de calcio.

Reconocer las facies de depósito y los rasgos de diagénesis en las rocas sedimentarias es fascinante porque éstas nos narran toda la historia tectónica y sedimentaria de una provincia geográfica, con las implicaciones geológicas y paleogeográficas siguientes: condiciones de clima, relieve flsiográfico, abundancia y diversidad de fauna y flora, variaciones y tipo de energía que controlaban a los sedimentos, profundidad del medio de 
depósito, composición química del ambiente, velocidad de sepultamiento de la columna estratigráfica, y cambios físico-químicos que ocurrían en el subsuelo.

El conocimiento de las rocas sedimentarias se inicia con el análisis de sus facies y de los ambientes de depósito que les dieron origen; consideraciones que sirven de fundamento para establecer las condiciones en las que las rocas se alteran durante su litificación y después de ésta. Particularmente, R.J. Dunhan (1963) postuló que las rocas pierden su porosidad debido a la precipitación del cemento calcítico durante el flujo del agua dulce o meteórica, entre los poros de las calizas; este proceso se lleva a cabo arriba del nivel freático, es decir en condiciones subaéreas. Dicha observación fue convincente para la comunidad geológica de aquel tiempo; ya que el fenómeno se observaba en los depósitos calcáreos del Pleistoceno que afloran en Bahamas, Florida y Cancún-Isla Mujeres. Más larde, los geólogos, además del martillo y la lupa, también utilizaron equipos de buceo, desde tanques individuales hasta submarinos capaces de recolectar muestras a profundidades mayores a los 3000 metros, es decir en el talud continental y en el fondo oceánico. El resultado de estas exploraciones fue sorpresivo, pues los sedimentos calcáreos no se cementan exclusivamente en ambientes subaéreos por el flujo del agua meteórica, como lo suponía R.J. Dunhan (1969); también se encontró que el proceso de cementación existe en ambientes marinos, profundos. Friedman (1964) reportó fangos de calcita microcristalina (micrita) completamente cementados a 75 metros de profundidad en el Mar Rojo; Milliman (1966) describe calcita con hábito cristalino fibroso (aragonita) cementando a las partículas de carbonato de calcio en antiguas islas volcánicas del Pacfflco; Gevirtz y Friedman (1966), reportaron cemento aragonítico en el fondo del Mar Rojo; Ginsburg et. al. (1967), y Land y Goreau (1970), encontraron a los arrecifes del Pleistoceno en Bermudas y Jamaica litificados con cemento marino de calcita hipermagnesiana; $y$, en fin, a partir de esos descubrimientos muchos investigadores han seguido localizando nuevas áreas marinas en las que se precipita cemento calcítico, en sus tres polimorfos: calcita con bajo contenido de magnesio, calcita hipermagnesiana (calcita con alto contenido de magnesio) y aragonita.

Los nuevos descubrimientos sobre diagénesis en calizas, suscitaron que un grupo importante y numeroso de investigadores se documentara sobre la formación de los constituyentes calcáreos, de su depósito y de las condiciones y tiempos de cementación. En este renglón, R.L. Folk identificó en 1973 tres medios mayores de cementación, los cuales se clasifican de acuerdo a: salinidad, temperatura, agitación y profundidad del agua, y por otro lado, a la profundidad y tiempo de sepultamiento de los sedimentos. Los procesos y ambientes diagenéticos mayores que propuso R.L. Folk son los siguientes: 


\section{A. DIAGÉNESIS POR FLUJO DE AGUA METEÓRICA}

a) Ambiente superficial: causado generalmente (aunque no necesariamente) por la reacción físico-química entre el agua dulce y la salobre con las calizas, que las transforma en suelos calcáreos o caliche; los cristales individuales resultantes de esta reacción son: calcita microcristalina (micrita), calcita en rombos y pisolitas de caliche (estructuras concéntricas mayores de $2 \mathrm{~mm}$ de diámetro con varios anillos) y calcreta o costras de calcita finamente laminadas.

b) Ambiente vadoso: la reacción entre el agua meteórica que se percola en el subsuelo, y las calizas, se realiza entre la superficie del terreno y el nivel freático; los productos calcíticos resultantes son cristales alongados y en bloques; los primeros son conocidos como cristales microestalactíticos. También existen minúsculos cristales de calcita del tamaño del limo, que Robert.J. Dunham (1963, 1969), los denominó limo calcítico, y que están asociados con las otras dos formas cristalinas.

c) Ambiente freático: la alteración química de la columna calcárea ocurre, como su nombre lo indica, bajo el nivel freático, y el producto de esta reacción es la precipitación de calcita espática en bloques y mosaicos.

Las formas de cementos calcíticos que se mencionan y que cristalizan a partir del agua meteórica saturada de carbonato de calcio, tienen un contenido bajo de sodio y magnesio, debido a que el agua dulce está infrasaturada de estos dos elementos. M.G. Gross (1964) analizó isotópicamente mediante oxígeno y carbono los cristales de calcita espática colectados de los ambientes diagenéticos en mención, y mostró que tenían una relación baja de $\mathrm{O}^{18} / \mathrm{O}^{16}$ y $\mathrm{C}^{13}$ / $\mathrm{C}^{12}$ (el agua de mar está enriquecida con el isótopo de oxígeno 18 y el de carbono 13, caso contrario al del agua dulce o meteórica): por lo que los análisis del autor reafirman las influencia meteórica en la estructuras cristalinas diagenéticas descritas.

\section{B. DIAGÉNESIS EN PERIMAREAS}

La zona de perimareas incluye la de intermareas, también conocida como zona litoral (limitada por las mareas diarias de pleamar y bajamar), y la de supramarea (superior a la de pleamar) que es inundada esporádicamente por las mareas vivas y durante las tormentas ciclónicas.

El nivel de salinidad que afecta a la zona de perimareas es sumamente variable, particularmente en la de supramarea, debido al flujo del agua meteórica al mar, originado por corrientes fluviales o por precipitación pluvial, que disminuye la salinidad; caso contrario sucede cuando el agua de mar se estanca en la zona de supramarea, pues la salinidad aumenta por evaporación hasta 8 veces más que el agua marina normal, que es de 35 partes por mil. El resultado de las fluctuaciones del nivel del mar y del flujo del agua meteórica es la precipitación de cementos complejos que varían en la zona de supramarea desde calcita en mosaicos y 
bloques, aragonita en fibras y calcita hipermagnesiana en bloques microcristalinos. R.N. Ginsburg (1953). J.C.M. Taylor y L.V. Ilings (1969). K.H. Wolf (1965) y otros autores, hicieron una revisión completa sobre el origen y las propiedades ópticas y químicas de los cementos mencionados, reconociendo que la pérdida de porosidad de los sedimentos calcáreos se efectúa rápidamente y que, al litificarse dan origen a las calizas sin necesidad de sufrir un gran sepultamiento; sucede lo contrario con las rocas que tienen partículas de feldespatos (derivados de la erosión de las rocas ígneas y metamórficas) que retardan más su litificación y pierden la porosidad intergranular en el subsuelo, por compactación o por cementación de arcilla precipitada "in situ", o bien, con calcita y sílice.

Las rocas que están en contacto con el agua de mar, en la zona de intramarea somera, pueden ser afectadas por el agua dulce que se infiltra a través de las partes del continente expuestas al mar, o sea, en la zona transicional freática marina y continental, en la que se precipitan cristales de calcita en fibras con zonas de crecimiento o maclas entrecruzadas y dolomita compuesta químicamente por carbonato doble de calcio magnesio, de la que posteriormente hablaremos debido a su importancia como producto de diagénesis.

\section{DIAGÉNESIS SUBMARINA}

Antes de los años sesenta se pensaba que los sedimentos calcáreos del fondo marino requerían ser sepultados para poder cementarse. Sin embargo, cuando se colectaron sedimentos de cuencas oceánicas como el Mar Rojo, se observó que éstos estaban litificados por calcita hipermagnesiana; sucedió lo mismo con los sedimentos del Océano Pacífico y en otras provincias marinas, como en el Golfo de California (Aguayo, 1984). que reportó calcita y dolomita litificando fangos de organismos pelágico, asociados con chimeneas hidrotermales. Frecuentemente, The American Association of Petroleum Geologists y The Society of Economic Paleontologists and Mineralogists preparan memorias especiales sobre mecanismos de cementación en sedimentos calcáreos, que enriquecen la editada por Bricker (1971).

\section{DIAGÉNESIS EN SUBSUELO}

Lo único que se conoce sobre mecanismos de cementación de calizas emplazadas a gran profundidad (varios cientos de metros), es que la calcita espática, como mineral cementante, es la más común, ya sea con hábito cristalino en bloques, en mosaicos, o con cristales individuales anhedrales y subhedrales (sin formas geométricas definidas 0 parcialmente desarrolladas); o bien. en forma de fibras y agujas, que se forman por recristalización a partir de la aragonita, preservándose los contornos cristalinos externos de los cristales originales de la aragonita y también el hábito microcristalino en bloque, de la calcita hipermagnesiana. El contenido de sodio y magnesio es bajo en todos los casos mencionados, ya que durante los procesos de 
recristalización hay movilización iónica de estos dos elementos, fuera de los cristales calcáreos.

R.L. Folk (1965) Y R.G.C. Bathurst (1971), realizaron una revisión profunda sobre los procesos de cementación y recristalización en las rocas y sedimentos calcáreos. El primero de ellos escribió su trabajo clásico Some Aspects of Recristalization in Ancient Limestones y el segundo publicó el libro Carbonate Sediments and Their Diagenesis. Ambos autores ( $y$ posteriormente muchos otros estudiosos del tema) concluyeron que el carbonato de calcio, en sus diferentes formas, es decir, como partículas individuales, matriz y cemento, es debido a la interacción de los siguientes factores: alta salinidad y evaporación por incremento en la temperatura del agua marina, por agitación e influencias orgánica, así como por la estabilidad del lecho marino que los contiene. Debido a los factores anteriores, el carbonato de calcio se precipita más rápidamente en la zona de intermareas que en los fondos oceánicos.

Por otro lado, el hábito cristalino que adquieren los diversos polimorfos de carbonato de calcio, dependen del grado de salinidad del agua, de la temperatura; y de la agitación del medio sedimentario; estos factores también se encargan de controlar que los elementos como por ejemplo, el sodio, magnesio, estroncio y otros queden atrapados en la estructura atómica cristalina de los minerales durante su formación.

En las últimas dos décadas se han escrito numerosos trabajos sobre los ambientes sedimentarios y los atributos diagenéticos que los caracterizan, debido, como ya dijimos, a que de sus análogos antiguos actualmente sepultados a varios kilómetros de profundidad, se siguen extrayendo grandes volúmenes de aceite crudo y de gas. Por este motivo, grupos de investigadores y de técnicos de diferentes disciplinas reinterpretan aquellas áreas que hace apenas pocos años eran consideradas "ampliamente" conocidas", a pesar de que las descripciones que se hacían de las rocas de la columna estratigráfica eran sumamente escuetas, como: "caliza macrocristalina de color gris, con vetillas de calcita y pirita diseminada". Estas descripciones de las rocas no eran suficientes para interpretar las condiciones del depósito de los sedimentos precursores a la roca, tal vez porque se consideraba que lo importante, a fin de cuentas, era localizar el petróleo sin prestar atención al tiempo y mecanismos de formación del mismo. Aparentemente esto es justificable si se toman en cuenta dos factores: primero, que habrán muchas estructuras geológicas relativamente someras por explorar, y segundo, que no se habla desarrollado profusamente la geoquímica del petróleo de manera que se carecía de criterios más precisos sobre la generación de hidrocarburos, y sobre los mecanismos de migración y de entrampamiento de los mismos. La corriente en la investigación actual es interdisciplinaria, encaminada al entendimiento científico para evaluar las provincias geológicas, y prospectar recursos naturales de interés económico. 


\section{DOLOMITA, MECANISMOS DE FORMACIÓN}

Otro paradigma fascinante en sedimentología y estratigrafía es el origen de la Dolomita (cristal rómbico de carbonato doble de calcio y magnesio).

Desde 1843 hasta la fecha, se han escrito no menos de 5000 trabajos sobre la dolomita y sus mecanismos de formación. ¿Por qué la comunidad científica le ha dedicado tantos esfuerzos? ¿Es éste un mineral raro en la naturaleza?, o bien, ¿será un recurso mineral valioso e insustituible?, ¡Nada de eso!, la dolomita es común en la columna estratigráfica; sin embargo, es un mineral que nos indica las condiciones físico-químicas y bioquímicas de los ambientes sedimentarios y de la historia evolutiva de la secuencia estratigráfica que la contiene, a pesar de que el proceso en sí tiende a destruir o a enmascarar los rasgos texturales y estructurales de la roca, y éstos pocas veces son identificables una vez que han sido alterados por el proceso diagenético de dolomitización. Entonces si sus efectos son destructivos ¿cómo podemos establecer o discernir la secuencia de evolución diagenética (paragénesis) de una roca o de una columna estratigráfica? Esta es una buena pregunta, que ha suscitado numerosos paradigmas entre los sedimentólogos $y$ estratígrafos.

La dolomita es abundante en la columna estratigráfica, no obstante, no lo es en los ambientes sedimentarios actuales. James D. Dana identificó en 1843 dolomitización en los complejos coralináceos de los atolones del Océano Pacífico, y postuló que se originaban en el mar debido a la reacción del ión de magnesio con el de carbonato de calcio contenido en los corales. Emest W. Skeats (1918) entre otros, asocia la abundancia de dolomita de los atolones del Pacífico con un reemplazamiento temprano a profundidades menores de 50 metros y en aguas cálidas. Anteriormente, en 1859, Sterry T. Hunt, señaló que la dolomita es consecuencia de la precipitación de sulfatos de calcio (yeso), que incrementa la relación magnesio/calcio en el agua, la solución resultante sobresaturada de magnesio reemplaza a las partículas sólidas de carbonato de calcio. Gilbert C. Cullis (op. cit.) y Emest W. Skeats (1905), encontraron cristales de dolomita con hábito cristalino en drusa, cubriendo cavidades en el complejo arrecifal de Funafuti en el Pacífico, a unos 200 metros de profundidad la consideraron como producto de precipitación primaria y no del reemplazamiento; estas investigaciones dieron origen a una nueva corriente sobre mecanismos de formación de mineral a que nos referimos. A partir de esos años, se han postulado no menos de 15 posibles orígenes de la dolomita, de las cuales sólo mencionaremos los más relevantes.

Ciertos organismos como las algas cianofíceas, tienden a atrapar entre sus filamentos soluciones ricas en magnesio y sintetizan y precipitan fotosintéticamente calcita hipermagnesiana que posteriormente recristaliza en protodolomita $y$ finalmente en dolomita; es decir que es una secuencia sucesiva de ordenación iónica entre el calcio, el magnesio y el carbonato dentro de la estructura atómica del mineral. 
La interpretación sobre el origen de la dolomita mediante este mecanismo ha sido tortuosa, debido a que actualmente no existen suficientes ambientes sedimentarios en donde se presenta. Mawson (1929) reportó dolomita margosa en sedimentos lagunares de Australia; esto fué confirmado por Alderman et al. en 1957. Ambientes sedimentarios actuales de supramarea con alta evaporación, en donde se precipitan evaporitas y dolomitas fueron descritas por Shinn (1964) en Florida; Shinn, Ginsburg y LLoyd (1965) en Bahamas; Illing, Wells y Taylor (1965) describieron las sebkhas o planicies de supramarea en el Golfo Pérsico; y en fin, muy pronto la teoría de la "supramarea" o de la "Sebkha" se convirtió en el dogma del siglo, para explicar los grandes depósitos de dolomitas presentes en la columna estratigráfica.

Los mecanismos físico-químicos dolomitizantes son diversos, la dolomita reemplaza a los cristales de calcita con una sobresaturación de iones de magnesio debido a alta evaporación del agua marina, precipitando también sulfatos de calcio, en forma de yeso y de anhidrita; la relación magnesio/calcio se incrementa cuando los iones de calcio son capturados iónicamente en la estructura cristalina de los sulfatos Esta teoría fue propuesta por Friedman y Sanders (1967) y Kinsman (1969) reafirmando la idea de S.T. Hunt, que la dió a conocer en 1859.

Otro origen inorgánico dolomitizante, es por la disminución de la salinidad en un ambiente inicialmente hipersalino (esquizohalino) al diluirse el agua marina normal con el agua meteórica (Folk y Land, 1974). La dolomita también se forma inorgánicamente cuando la solución acuosa enriquecida en iones de magnesio, migra por gravedad y se infiltra entra los sedimentos calcáreos no consolidados (Deffeyes, Lucia y Weyl, 1965).

El mecanismo contrario existe cuando las soluciones saturadas de calcio y magnesio fluyen hacia arriba por capilaridad durante la evaporación del agua, y las partículas calcáreas se dolomitizan; a este proceso se le conoce como teoría capilar (Hsu y Siegenthaler, 1969). Goodell y Garman (1969) proponen un mecanismo de disolución y sobrecrecimientos en los cristales de dolomita previamente formados para dar lugar a otros más grandes (agradación diagenética). Hanshaw et al. (1971), Land (1973) y Land et al. (1975) sugieren que los procesos de dolomitización son más efectivos en la zona freática marina, o sea, en la zona en la que el agua meteórica infiltrada en las calizas, se mezcla con el agua marina.

El agua del mar es una de las fuentes principales de iones de magnesio otra es la arcilla, conocida como esmectita sódica, enriquecida en iones de magnesio, sodio, hierro y otros constituyentes. La arcilla durante su compactación libera al magnesio por incremento de temperatura y dolomitiza a los sedimentos calcáreos que contienen a la arcilla; tal es el caso en la costa de Louisiana en los Estados Unidos $y$ en los sedimentos recientes depositados en las cuencas oceánicas. Los procesos de dolomitización descritos hasta este momento, ocurren casi simultáneamente o un poco después del depósito de los sedimentos 
bajo condiciones superficiales o someramente sepultados.

La dolomitización también se presenta durante el sepultamiento de los sedimentos calcáreos y al producto se le denomina dolomita "profunda" y cuando ésta se asocia con la deformación estructural y el fracturamiento de la columna estratigráfica, se le conoce como dolomita "tectónica". En ambos casos, la arcilla insoluble, ya sea de origen orgánico o inorgánico diseminada en las calizas, libera los iones de magnesio cuando se somete la caliza bajo presión, recristalizándose y disolviéndose parcialmente. Los iones liberados se movilizan junto con el agua intersticial contenida en los horizontes arcillosos que están intercalados con las calizas.

Otra teoría que intenta explicar los grandes espesores de rocas dolomitizadas, es aquella propuesta por J.E. Adams y ML. Rhodes, en 1960. Los autores explican que hacia el subsuelo los fluidos sobresaturados en magnesio (provenientes éstos de las evaporitas, sulfatos y cloruros) más superficiales se infiltran en las calizas, reemplazándolas por dolomita. Al proceso diagenético se le conoce como teoría del reflujo.

Existe otro proceso de dolomitización por soluciones saturadas de magnesio y calcio, asociadas con aguas geotérmicas o hidrotermales a grandes profundidades; estas soluciones se precipitan como cualquier otro cemento, en las fracturas o en otras cavidades disponibles en la roca, denominándose dolomita primaria; o sea, que no es producto de reemplazamiento sino de la precipitación primaria del fluido saturado de magnesio, calcio y bicarbonato. En este caso la temperatura, anómalamente alta, sustituye al tiempo de reemplazamiento bajo condiciones normales $\left(25^{\circ}\right.$ a $\left.50^{\circ} \mathrm{C}\right)$.

Como se hizo mención anteriormente, una vez que ocurre el reemplazamiento de la calcita por la dolomita, la textura original de la roca se pierde y no es posible identificarla con el microscopio petrográfico convencional. Lo anterior constituye un problema crítico para los sedimentólogos y estratígrafos, ya que dependen del reconocimiento de los constituyentes petrográficos originales, para poder interpretar el ambiente primario de sedimentación de la roca, su evolución físico-química y mineral durante su litificación y de la historia tectónica de la provincia geológica para predecir, en el subsuelo, horizontes estratigráficos de interés económico.

La necesidad que tienen los científicos de identificar los rasgos primarios de las rocas calcáreas dolomitizadas, los indujo a experimentar con varias técnicas de investigación; en un principio, éstas fueron simples pues utilizaron reactivos de composición orgánica como la alizarina roja y otras similares, para distinguir entre la calcita y la dolomita (Friedman, 1959); lo cual es posible, ya que la primera se tiñe de rojo al reaccionar con la solución y la segunda permanece incolora. Con este método es posible identificar ambos minerales, pero no se revela la textura original de la roca sedimentaria. En 1965, R.F Slppel y 
E.D. Glover resaltaron algunos rasgos primarios en calizas recristalizadas, pero no en dolomitas, por medio de la luminiscencia (luminosidad) diferencial que se produce en los minerales cuando se excitan sus iones durante el bombardeo de electrones que inciden sobre la superficie de la preparación rocosa. También se han utilizado otros métodos para producir la luminosidad de los componentes calcáreos; entre ellos están la excitación iónica mediante rayos $X$, $y$ el método de termoluminiscencia que activa, por medio de calor, algunos iones atrapados en la red cristalina del mineral.

Los métodos descritos tienen dos desventajas, primero, que dañan la preparación en lámina delgada de la roca, y segundo, que la operación de la instrumentación no es fácil, ya que se requiere de gran experiencia y paciencia para obtener resoluciones aceptables. Recientemente en 1985, J.J. Dravis y D.A. Yurewicz de la Compañía Petrolera Exxon de Estados Unidos, obtuvieron magníficos resultados al lograr hacer resaltar los rasgos texturales primarios de las rocas dolomitizadas, utilizando una fuente de luz fluorescente adaptada al microscopio petrográfico convencional. La aportación de dichos autores es más que satisfactoria ya que la muestra de roca en lámina delgada, puede ser observada un sinnúmero de veces, sin sufrir daño alguno. El empleo del método de fluorescencia en rocas dolomitizadas, abre un nuevo campo para la interpretación de la evolución de las provincias geológicas calcáreas.

En épocas anteriores. sin estos avances científicos, poco se podía investigar acerca de las columnas estratigráficas de calizas recristalizadas y dolomitizadas. La aplicación del método analítico de fluorescencia permite identificar tanto los constituyentes orgánicos e inorgánicos, como la textura original de la roca; además pueden distinguirse los diversos cementos y su tiempo relativo de precipitación y se observan los procesos evolutivos de la porosidad, así como la geometría y distribución de la misma (ver lámina 1). D.H. Zenger, J.B. Dunham y R.L. Ethington, en 1980, editaron un compendio sobre conceptos y modelos de dolomitización, en el cual resumen los principales logros científicos actuales al respecto.

Los avances que se han logrado en la Sedimentología y en Estratigrafía dependen en gran parte de la utilización de la electrónica para resolver problemas geológicos, puesto que no sólo se pueden identificar los parámetros primarios y secundarlos de las rocas, sino también cuantificarlos.

\section{DIAGÉNESIS EN ARENISCAS}

La investigación sobre el origen de los sedimentos terrígenos es amplia, particularmente en lo referente a los mecanismos de transporte y acumulación en ambientes tales como aluviales, fluviales, eólicos, litorales, marinos someros y profundos etc. Sin embargo, la información que se tiene sobre los procesos diagenéticos que actúan en este tipo de sedimentos, es comparativamente más escasa que en las calizas; concretamente, cuando se trata de 
explicar el origen y tiempo de formación de la porosidad en areniscas, y de la destrucción de las partículas primarias por sepultamiento de la columna estratigráfica.

Antes de 1975 la comunidad sedimentológica consideraba que más del $90 \%$ de la porosidad contenida en los terrígenos, era primaria-intergranular, es decir con huecos formados durante el depósito de las partículas sedimentarias y de origen secundario cuando la roca se fractura.

A principios de la década de los setenta algunos geólogos rusos habían observado aisladamente cantidades significativas de porosidad en areniscas, debido a la disolución del mineral cementante y de algunos litoclastos; aunque no fué sino después de la segunda mitad de esa década cuando se publicaron numerosos trabajos que describen la importancia de los procesos de diagénesis en el subsuelo, como generadores de la porosidad secundaria en areniscas; ejemplos de ellos son los trabajos de: V. Schimdt, 1976; G.D. Stanton y E.F. McBride, 1976 E.D. Pittman, 1979; V. Schlmidt y DA McDonald, 1979 y muchos otros más.

La aportación más importante a la Sedimentología y a la Estratigrafía que hicieron los investigadores mencionados y otros no referidos, fue el hecho de reconocer cuando menos cinco tipos diferentes de porosidades secundarias en areniscas, y que se les puede diferenciar según el proceso de formación y a la relación textural que tienen con el resto de la roca. Estos cinco tipos son los siguientes, según su origen:
1) por fracturación,

2) por contracción de las partículas individuales, de la matriz o del cemento,

3) por disolución selectiva de las partículas,

4) por disolución selectiva del cemento o de la matriz, y

5) por disolución selectiva de los minerales autigénicos precipitados en el subsuelo.

La identificación de los diferentes tipos de porosidad en las areniscas, así como de los minerales de neoformación o autigénicos, que se forman en el subsuelo, son importantes índices geológicos para predecir recursos minerales, ya que la disolución de algunos minerales y la precipitación de otros, depende de la presión y la temperatura a la que están sometidos, de su composición química, de los fluidos intersticiales y del tiempo geológico en el cual actúan los procesos mencionados. En el caso particular de muchos campos de aceite y de gas, las porosidades secundarlas en los estratos de rocas terrígenas, juegan un papel importante en la formación de reservorios de hidrocarburos; como ejemplos se mencionan aquellos yacimientos estudiados por J.B Hayes (1979) y E.D. Plttman (1979). A pesar de los grandes avances que se han hecho desde la segunda mitad de la década anterior, el estudio y el entendimiento pleno de la diagénesis en areniscas, aún está en ciernes y los investigadores están encausando la investigación descriptiva y cuantitativamente, a este respecto. Esto es evidente en las publicaciones de D.A. 


\section{LÁMINA No. 1}

a) Fotomicrografía de una caliza dolomitizada, tomada en lámina delgada con el microscopio petrográfico convencional. Por este método no se revela la textura original que tenía la roca antes del reemplazamiento. Longitud de la barra $=500$ micras.

b) La misma que la anterior y a igual escala (flechas como referencia). Fotomicrografía tomada con fluorescencia de rayos $X$, revelando la composición y textura originales de la roca antes de ser reemplazada por dolomita. Las partículas mayores son oncolitos, es decir, estructuras concéntricas de origen algáceo cementadas con calcita y pellas fecales. Longitud de la barra $=500$ micras. 


\section{LAMINA 1}
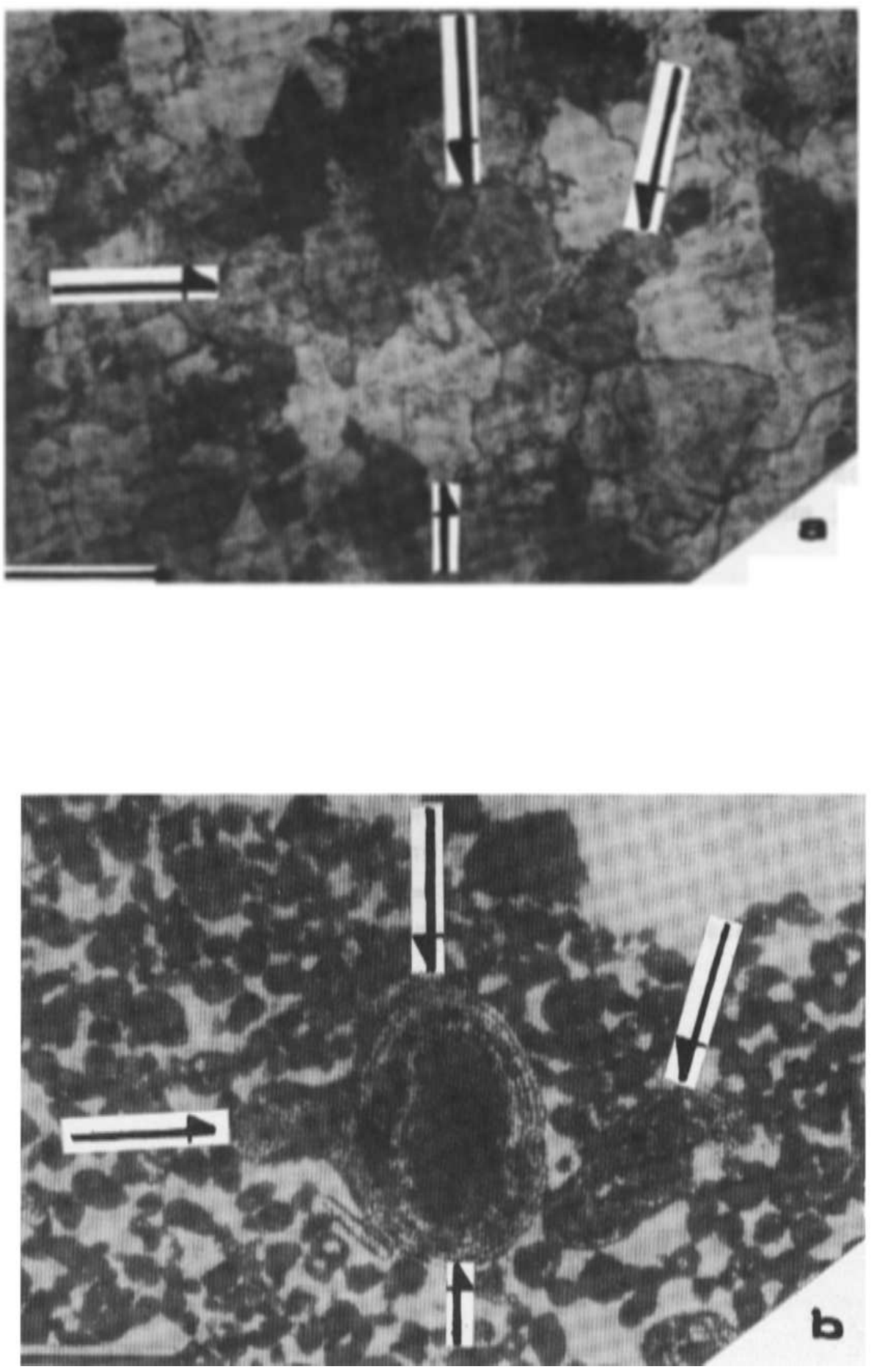


\section{LÁMINA NO. 2.}

a) Fotomicrografía de una arenisca constituida por partículas angulares y subangulares de cuarzo (8102), Con baja porosidad intergranular debido a su aparente alta compactación. La muestra fue tomada en $16 \mathrm{~m} /$ na delgada con el microscopio petrogrétlco convencional. Longitud de barra $=500$ micras.

b) La misma que la anterior tomada con luz catodoluminiscente y a igual escala (flechas como referencia). Nótese que las partículas tienden a la esfericidad y a la redondez, opuesto a lo que se aprecia en el método convencional (e). El proceso revela que las partículas han crecido por cementación sintaxial (color negro), o sea, en continuidad óptica con el eje de elongación. Longitud de la barra $=500$ micras. Foto tomada de : The American Association of Petroleum Geologists memoir 28, (1979). 


\section{LAMINA ?}
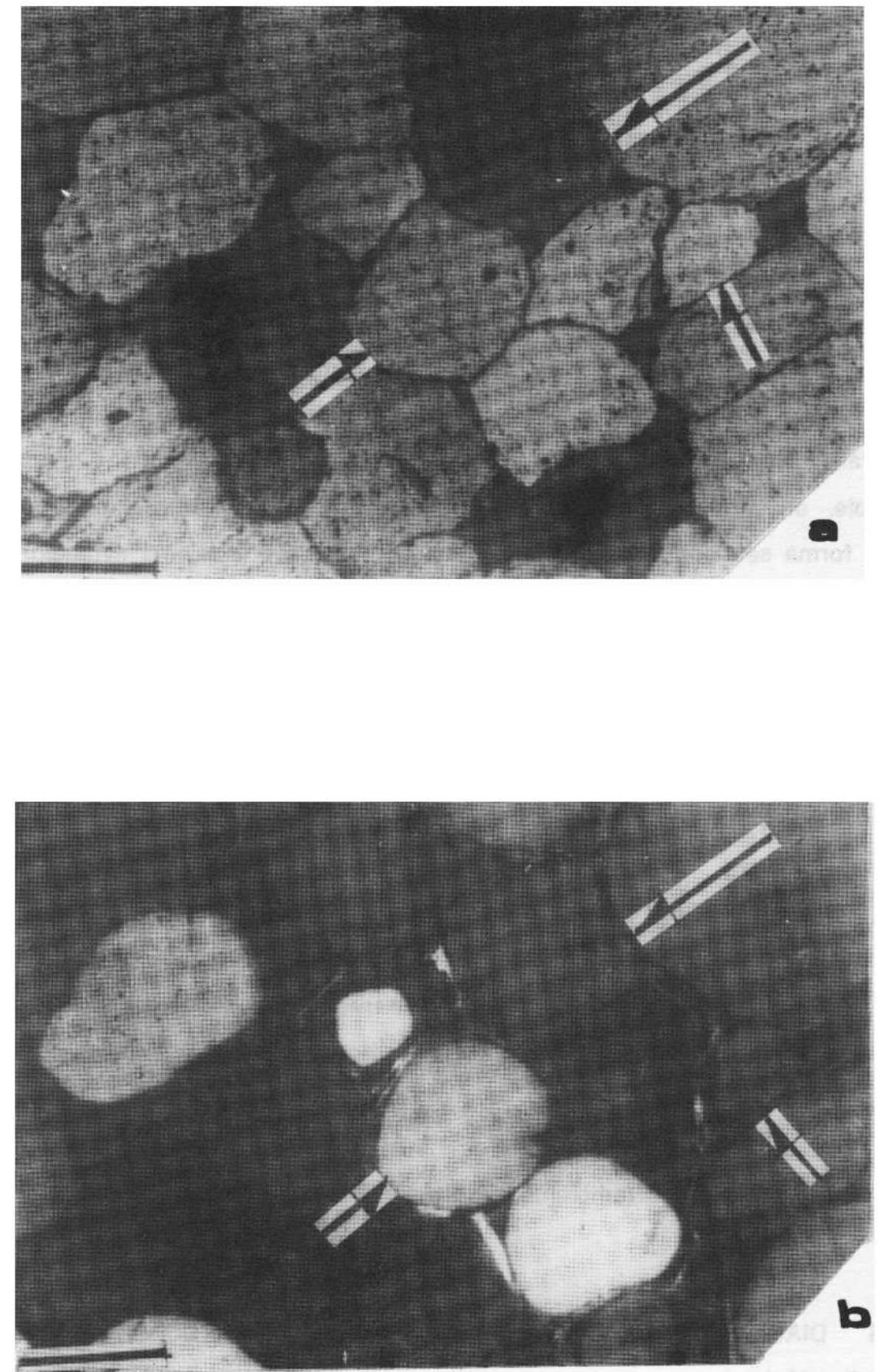
McDonald y R.C. Surdam (1984), así como en la de J.E. Welton, publicada en el mismo año. Ambos trabajos asocian los atributos texturales de las rocas con su composición mineral y química, por medio de métodos analíticos determinativos con la microsonda de barrido y difracción de rayos $X$, previa observación bajo el microscopio electrónico de barrido y con el petrográfico convencional (ver lámina 2).

La sistematización y cuantificación de los datos geológicos, geofísicos y geoquímicos nos conducen a la evaluación de grandes provincias geológicas, y para ello se ha hecho necesario compilar, integrar e interpretar toda la información posible, en forma interdisciplinaria. Por ejemplo, una forma secuencial para resolver problemas de evolución tectónica, sedimentación y diagénesis sería el siguiente:

\section{SÍNTESIS METODOLÓGICA PARA EL ESTUDIO DE PROVINCIAS GEOLÓGICAS}

1. DATOS ESTRATIGRÁFICOS $Y$ ESTRUCTURALES.

\section{ANÁLISIS DE FACIES SEDIMENTARIAS (clase} y distribución).

3. PROVINCIA SEDIMENTARIA (ambiente $y$ subambientes).

\author{
4. ATRIBUTOS DIAGENÉTICOS (variación, \\ asociación y distribución).
}

\section{EVOLUCIÓN TECTÓNICA (estable,} subsidencia, emersión, divergencia, convergencia).

6. PARAGÉNESIS (tectonismo, sedimentación y diagénesis secuencial).

\section{PREDICCIÓN DE RECURSOS MINERALES.}

Los análisis sedimentológicos y estratigráficos, al igual que otras ciencias geológicas, geoquímicas y geofísicas tienen como objetivo, proporcionarnos una mejor comprensión de este gran sistema dinámico y cambiante en el que vivimos, llamado planeta Tierra, que es el producto de toda una evolución de los procesos físico-químicos y biológicos que la rigen y a los que rige, y que difícilmente podrían ser representados matemáticamente ya que son numerosas las variables geológicas que intervienen en el Espacio y el Tiempo.

\section{BIBLIOGRAFÍA}

Adams, J,E., y M.L. Rhodes.. "Dolomitization by seepage refluxion", Boletín de The American Association of Petroleum Geologists, Vol. 44, pp. 1912-1920, 1960.

Aguayo, C.J.E.,. "Estudio de los sedimentos terrígenos de la Cuenca de Guaymas, Golfo de California, Noroeste de México", en Revista del Instituto Mexicano del Petróleo, Vol. XVI 4, pp. 5-35, México, 1984

Alderman, A.R., H.Skinner., W. Catherine,. Dolomite sedimentation in the southeast of South Australia, en 
Boletín de American Journal of Science, Vol. 255 p. 581-587, 1957.

Armstrong. R.L., Control of sea level relative to the continents. Nature, Vol. 221, 1042-1043, 1969.

Bathurst, R.G.C., "Diagenetic fabrics in some British Dinantian limestones". Liverpool and Manchester Geological Journal, Vol. 2, pp. 11-36, 1958,

,. "Diagenesis in Mississippian Calcilutites and pseudobreccias". En Boletín de Journal Sedimentary Petrology, Vol. 29, 365-376, 1959.

,. "Carbonate Sediments and their Diagenesis. Developments in Sedimentology", No. 12. Elsevier Publ. Co., pp. 1-620, 1971.

Bergenback, R.E. y R.T.Terriere, "Petrography and petrology of Scurry Reef, Texas". En Boletín de The American Association of Petroleum Geologists, Vol. 37, pp. 1014-1029, 1953.

Bricker, O.P., "Carbonate Cements" en Studies in Geology, No. 19, Johns Hopkins Univ., pp. 1-376, 1971. Cullis, C.G., "The mineralogical changes observed in the cores of the Funafuti bearings", en Section XIV Royal Society of London, 392-420, 1904.

Dana, J.D., "On The analogies between the modern igneous rocks and the so called primary formations, and the metamorphic changes produced by heat in the associated sedimentary deposits", en Boletín de The American Journal of Sciences, 45, 104-129, 1843.

Deffeyes, K.J., F.J. Lucia y P.K. Weyl, "Dolomitization of Recent and Plio-Pleistocene by marine evaporite waters on Bonaire Netherlands Antilles", en Dolomitization and Limestone Diagenesis, The Society of Economic Paleontologists and Mineralogists, Special Publication No. 13, 71-88, 1965.
Dietz, R.S., "Continent and ocean basin evolution by spreading of the sea floor", en Nature, Vol. 190,pp. 854-857, 1961.

Dravis, J.J.y D.A. Yurewicz, "Enhanced carbonate petrography using fluorescence microscopy", en Journal of Sedimentary Petrology Vol. 55, No. 6, 795-804, 1985.

Dunham, R.J., "Classification of carbonate rocks according to depositional textures", en Classification of Carbonate Rocks, The American Association of Petroleum Geologists. Ham W, (ed), Memoir I, pp. 108-121, 1962.

, "Early vadose silt in Townsend Mound (Reef), New Mexico (abs.)", en Boletín de The American Association of Petroleum Geologists, Vol. 47, 356, 1963.

"Early vadose silt in Townsend Mound (reef) New Mexico", en Depositional Environment in Carbonate Rocks. Society Economic Paleontologists and Mineralogists, Special Publication, 14,139-181, 1969.

Folk, R.L., "Practical petrographic classification of limestones", en Boletín de The American Association of Petroleum Geologists, 43, 1-38, 1959.

,' "Spectral subdivision of limestone types., en Classification of Carbonate Rocks, The American Association of Petroleum Geologists, Ham W, (ed), Memoir I, pp. 62-84, 1962.

_, "Some aspects of recrystallization in ancient limestones-, en Dolomitization and Limestone Diagenesis, The Society of Economic Paleontologists and Mineralogists. Special Publication, 13, pp. 14-48, 1965.

, "Carbonate Petrography in the Postsorbian age", en Evolving Concepts in Sedimentology. (Ed). R.N. Ginsburg. The Johns Hopkins University Studies in Geology No. 21, pp. 118-158,1973. 
_ y L.S. Land, 1974,Mg/Ca ratio and salinity: Two controls over crystallization of dolomite, The American Association of Petroleum Geologists,59, 60-68.

Friedman, G.M. y J.E. Sanders, 1967, Origin and occurrence of dolostones, en Carbonate Rocks Developments in Sedimentology 9, Elsevier Publ. Co pp. 167-348.

1959, Identification of carbonate minerals by staining methods, Journal of Sedimentary Petrology, 29, 87-97.

1964, Early diagenesis and lithification in carbonate sediments, Journal of Sedimentary Petrology, 34, 777-813.

Gevirtz, J.L., y G.M. Friedman, 1966, Deep sea carbonate sediments of the Red Sea and their implications on marine lithification, Journal of Sedimentary Petrology, 36, 143-151.

Ginsburg, R.N, "Beachrock in South Florida", en Boletín de Journal of Sedimentary Petrology, Vol. 23, pp. 85-92., 1953.

E.A., Shinn y J.H. Schroeder, "Submarine cementation and internal sedimentation within Bermuda reefs (abs.)", en Geological Society of America, Special Paper 115, pp. 78-79, 1967.

Goodell, H.G., y R.K. Garman, "Carbonate geochemistry of Superior deep test well, Andros Island, Bahamas", en Boletín de The American Association of Petroleum Geologists, Vol. 53, pp. 513-536, 1969.

Graf, D.L., y J.E. Lamar, "Petrology of Fredonia oolite in Southern Illinois", en Boletín de The American Association of Petroleum Geologists, Vol. 34, pp. 2318-16, 1950.
Gross, M.G, Variation in the $\mathrm{O}^{18} / \mathrm{O}^{16}$ and $\mathrm{C}^{13} / \mathrm{C}^{12}$ ratios of diagenetically altered limestones in the Bermudas islands", en Journal of Geology, Vol. 72, 170-194., 1964. Hanshaw, B.B., W. Back, y R.G. Deike, "A geochemical hypothesis for dolomitization by groundwater", en Economic Geology, 66, 710-724, 1971.

Hayas, J.B., "Sandstone diagenesis-The hole truth", en Aspects of Diagenesis, The Society Economic Paleontologists and Mineralogists, Special Publication no 26, 127-140, 1979.

Hess, H.H. "History of ocean basins", en Petrologic studies, Buddington Volume, Geological Society of America, 599-620, 1962.

Hsú, K,J. y C. Siegenthaler, "Preliminary experiments on hydrodynamic movement induced by evaporation-their bearing on the dolomite problems", en Sedimentology 12 , 11-26, 1969.

Hunt, S.T., "On some reactions of the salts of lime magnesium, and the formation of gypsums and magnesium rocks", en American Journal of Science, Vol. 28, No. 2, pp. 377-381, 1859.

Illing, L.V., A.J., Wells y J.C.M. Taylor, "Penecontemporary dolomite in the Persian Gulf", en Dolomitization and limestone Diagenesis The Society Economic Paleontologists and Mineralogists, Special Publication, 13, pp. 89-111, 1965.

Kinsman, D.J.J, "Modes of formation sedimentary associations and diagnostic features of shallow-water and supratidal evaporitics", en Boletín de The American Association of Petroleum Geologists, No.53, pp. 1830-840, 1969.

Land, L.S., y T.F. Goreau, "Lithification of Jamaican reefs", Journal of Sedimentary Petrology, Vol. 40, pp. 457-462, 1970. 
_ , "Holocene meteoric dolomitization of Pleistocene Limestones, North Jamaica", en Sedimentology, Vol. 20, pp. 411-424, 1973.

M.K.I. Salem y P.W. Morrow, "Paleohydrology of ancient dolomites: Geochemical evidence", The American Association Petroleum Geologists, Vol. 59, pp. 1602-1625, 1975.

Mawson, D., "South Australian algal limestones in the process of formation". en Quarterly Journal Geological Society of London, Vol. 85, pp. 613-621, 1929.

McDonald, DA Y R.C., Surdam (ed), "Clastic Diagenesis", en The American Association Petroleum Geologists Memories, 37, 1-434, 1984.

Menard, H.W., Marine Geology of Pacific. New York. Mc. Graw Hill, pp. 1-271, 1964.

Millman, J.E., "Submarine lithification of carbonate sediments", Science, Vol.153, pp. 994-997, 1966.

Pittman, E.D., "Porosity diagenesis and capability of sandstones reservoirs", en Aspect of Diagenesis. The Society of Economic Paleontologists and Mineralogists. Special Publication 26, pp. 15-174, 1979.

Schmidt, V., "Secondary porosity in the Parsons Lake Sandstones (abs)", en Geological Society of Canada and Mineral, Annual meeting. Vol. p. 1, 50, 1976.

y. D.A., McDonald, "The role of secondary porosity in the course of sandstone diagenesis", en Aspects of Diagenesis. The Society of Economist Paleontologists and Mineralogists, Special Publication 26, pp. 175-208, 1979.

Shinn, E.A., "Recent dolomite, Sugarloaf Key, Florida", en South Florida Sediments Guidebook Field Trip 1, Geological Society of America Annual Convention, pp. 26-33, 1964.

_ R.N. Ginsburg, and R.M. Lloyd, "Recent supratidal dolomite from Andros Island, Bahamas", en
Dolomititation and Limestones Diagenesis, The Society of Economics Paleontologists and Mineralogists. Special Publication 13, pp. 112-123, 1965.

Sippel. R.F. y E.D. Glover, "Structures in carbonate rocks made visible by luminescence petrography", en Science, Vol. 15, 1283-1297, 1965.

Skeats. E.W., "On the chemical and mineralogical evidence as the origin of the dolomites of Southern Tyrol", en Geological Society, Quarter Journal London Vol. 61, pp. 97-141, 1905.

"The formation of dolomite and its bearing on the coral reef problem", American Journal of Science, Vol. 45, pp. 188-189, 1918.

Sorby, H.C., "On the microscopical structure of some British Tertiary and post-Tertiary fresh-water marls and limestones", Geological Society London Quarter Journal 9, pp. 344-346, 1853.

Stanton, G.D. y E.F. McBrlde, "Factors influencing porosity and permeability of Lower Wilcox (Eocene) Sandstone, Karne. Country, Texas" (abs), en The American Association of Petroleum Geologists, Annual meeting, Vol. 1, p. 119, 1976.

Taylor J.C.M. y L.V. Illing, "Holocene intertidal calcium carbonate cementation, Qatar. Persian Gulf" Sedimentology, Vol. 12, pp. 69-107, 1969.

Walker, R.G., "Mapping up the turbidite mess, en Evolving Concepts in Sedimentology, Ed R. Ginsburg. The Johns Hopkins Univ. Studies in Geology, No. 21, pp. 1-37, 1973.

Welton,J.E., SEM Petrology Atlas, en Methods in Exploration Series of The American Association of Petroleum Geologists, pp. 1-237, 1984.

Wolf, K.M. y G.V. Chilingarian, "Diagenesis of Sandstones and Compactation", Developments in Sedimentology 18B Elsevier Publ. Co. pp. 1-808, 1976. 
Wolf, K.H., "'Gradational sedimentary products of calcareous algae", en Sedimentology, Vol. 5, pp. 1-37, 1965
Zenger, D.H. J.B. Dunham y R.L. Ethington “, Concepts and models of Dolomitization", en The Society of Economic Paleontologists and Mineralogists, Special Publications No. 26, pp. 1-320, 1960. 